\title{
Scaling and chaos in periodic approximations to the two-dimensional Ising spin glass
}

\author{
David A. Huse \\ Physics Department, Princeton University, Princeton, NJ 08544 \\ Lee-Fen Ko \\ SpecTran Corporation, 50 Hall Road, Sturbridge, MA 01566
}

(March 12, 2018)

\begin{abstract}
We approximate a two-dimensional spin glass by tiling an infinite lattice with large identical unit cells. The interactions within the unit cell are chosen at random, just as when one studies finite-size systems with periodic boundary conditions. But here the unit cells are instead connected to form an infinite lattice, so one may examine correlations on all length scales, and the system can have true phase transitions. For such approximations to the Ising spin glass on the square lattice, we apply the free-fermion method of Onsager and the anticommuting operator approach of Kaufman to obtain numerically exact results for each realization of the quenched disorder. Each such sample shows one or more critical points, with the distribution of critical temperatures scaling with the unit cell size, consistent with what is expected from the scaling theory of low-dimensional spin glasses. Due to "chaos", the correlations between unit cells can change sign with changing temperature. We examine the scaling of this chaos with unit cell size. Many samples have multiple critical points due to the interactions between unit cells changing sign at temperatures within the ordered phases.
\end{abstract}

\section{INTRODUCTION}

The numerical investigation of the equilibrium, low-temperature properties of finite-dimensional spin-glasses has relied heavily on studies of models on finite-sized lattices, usually with periodic boundary conditions. [1 \&] The data for finite lattices are extrapolated to the infinite lattice limit, typically using scaling assumptions. In this paper we explore a somewhat different approach to approximating an infinite two-dimensional Ising spin glass. Instead of putting periodic boundary conditions on the finite-size spin glass sample, we instead "tile" the infinite lattice with identical unit cells, each containing the same realization of the random exchanges. For planar lattices this produces a two-dimensional Ising model, various properties of which can be obtained to numerical precision using algorithms whose time and memory requirements grow only as a power of the area of the unit cell. In particular, we examine the square lattice model with only nearest-neighbor interactions that are independently and identically distributed according to a continuous probability distribution.

What should we expect for the behavior of this system? For higher temperature, when the correlation length is less than the unit cell size, the periodicity of the lattice is not relevant and the behavior should be that of a spin glass. However, when the correlation length exceeds the unit cell size, the behavior should cross over to that of a specific periodic Ising model, which will have a phase transition in two dimensions. The resulting ordered phase may be ferromagnetic between unit cells, so the local magnetization patterns in each unit cell are identical and aligned, or antiferromagnetic, so adjacent unit cells have opposite local magnetization patterns. In either case, the magnetization pattern within a unit cell is that of a spin glass: a random pattern that is determined in all its details by the temperature and the specific quenched random interactions in that sample. By calculating the spectrum of each specific sample's transfer matrix, we can locate its phase transitions and determine whether they are into ferro- or antiferromagnetic phases. Even in the disordered phase, we can determine whether the correlations between adjacent unit cells are more ferro- or antiferromagnetic by comparing the gaps in the spectrum at the center and edge of the Brillouin zone.

For each sample, we obtain the spectrum of its transfer matrix, and thereby the correlation lengths for ferro- and antiferromagnetic ordering. The phase transitions are located by finding where the gap below the highest "level" in the spectrum vanishes, corresponding to an infinite correlation length. Some samples show multiple phase transitions, because the effective interaction between adjacent unit cells changes sign with changing temperature. We identify the highest transition temperature as $T_{c}$. In the limit of an infinite unit cell, we have the two-dimensional spin glass that has $T_{c}=0$ and a correlation length that behaves as $\xi \sim T^{-\nu}$ at low temperature $T$. But for unit cells of linear size $L$, the system "realizes" that it is periodic when the correlation length exceeds $L$, so it is reasonable to expect that $T_{c} \sim L^{-1 / \nu}$. We measure the distributions of $T_{c}$ by examining many samples for each value of $L$ we study, and find that the distributions do scale with $L$ as expected, with $\nu \cong 2.7$.

The more novel and striking results of our study are those addressing the so-called "chaos" in the spin-glass correlations. The effective interaction between two well-separated spins in a spin-glass is due to many competing 
(frustrated) chains of interaction through intermediate spins. The precise balance between the energetic and entropic contributions to these interactions changes with changing temperature. The net result of this is that the sign of the correlation between the two distant spins can change as the temperature is changed. 99.10] In our specific geometry this can be readily seen as the sign of the correlations between equivalent spins in different unit cells of a specific sample changing between ferro- and antiferromagnetic as the temperature is changed. When these sign changes occur below $T_{c}$ they cause additional phase transitions, since they change the nature of the ordered phases between ferroand antiferromagnetic. This is seen in many samples. We can also detect the sign changes when they occur above $T_{c}$ by monitoring which of the two correlation lengths (ferro- or antiferromagnetic) is longer. Our new numerical approach to this problem allows a much more direct study of this temperature-driven chaos than was possible in the previous Monte-Carlo study by Ney-Nifle and Young. [8] We find that the number of sign changes grows rapidly with increasing unit cell size, as is expected from scaling arguments.

\section{THE MODEL AND THE METHOD}

We consider an infinite square lattice Ising model tiled by a regular square pattern of square unit cells of size $L \times L$ spins, $L$ even. We take the pattern of quenched random interactions in the upper $L \times(L / 2)$ half of the unit cell to be the mirror image of that in the lower half produced by reflecting about a horizontal line passing through the central row of spins; this makes the the transfer matrix that takes one vertically across a row of full unit cells Hermitian and hence easier to handle numerically. The reduced bond strength of the nearest-neighbor bond between sites $i$ and $j$ is $K_{i j}=J_{i j} / T$, where $J_{i j}$ is a random number drawn from a distribution that is uniform on the two intervals $(-1.6,-0.4)$ and $(0.4,1.6)$, and $T$ is the temperature. In general, this bond is duplicated in the other half of the unit cell and in all other unit cells throughout the infinite lattice. The unusual bond strength distribution we use was chosen because the algorithm has difficulty with both very strong and very weak bonds. However, since the distribution is broad and continuous, we expect behavior similar to that for more standard continuous distributions, such as the Gaussian, that include weak bonds.

The partition function $Z=V^{M}, M \rightarrow \infty$, where $V=W W^{\dagger}$ is the transfer matrix across a row of full unit cells and $W$ is that across a row of half unit cells. The transfer matrix $W$ is a product of row-to-row transfer matrices of horizontal bonds, $V_{h}$ 's, and vertical bonds, $V_{v}$ 's. The $V_{h}$ 's and $V_{v}$ 's have the usual quadratic form in terms of the anticommuting operators, 11 14 except that the couplings vary from site to site, both within and between rows. Note that as $K_{i j} \rightarrow-K_{i j}, K_{i j}^{*} \rightarrow K_{i j}^{*}+i \pi$. The quadratic form allows the transfer matrices to be represented as matrices, $R$ 's, in the space of anticommuting operators, which is of dimension twice the width of the lattice. This transforms a problem exponential in the size of the lattice to one that is linear. In other words, the problem of finding the eigenvalues, $\Lambda_{i}$ 's, of the transfer matrix is transformed to that of computing the energy levels of the free fermions. In this free-fermion representation, the eigenvalues of the transfer matrix come in pairs of $\exp \pm \lambda_{i}(\omega), i=1, \ldots, L$, for each spatial frequency $\omega \in(-\pi, \pi)$; the energy levels of the free fermions are simply $\pm \frac{1}{2} \lambda_{i}$ 's. In the ground state, all negative energy levels are filled. The first excited state consists of an excitation at the gap where $\lambda_{1}$ is minimum. Thus the correlation length $\xi$ along the vertical direction in units of the unit cell height is $\left[\min _{\omega} \lambda_{1}(\omega)\right]^{-1}$. The special structure of the eigenvalues is the result of each of the $R$ 's being a direct product of two-dimensional rotations.

The algorithm consists of multiplying the $2 L \times 2 L$ matrices $R(\omega)$ 's from each row of horizontal and vertical bonds through $L / 2$ rows, then multiplying by its hermitian conjugate for the mirrored half of the $L / 2$ rows. The gap is then computed from the smallest eigenvalue above one. Because the entries in these matrices are hyperbolic functions, high precision is required for any reasonable size unit cell; we used an arbitrary precision software [15] to do the computation.

More details about calculating the properties of Ising models with inhomogeneous unit cells will be reported elsewhere. 16]

\section{PHASE TRANSITIONS}

For each sample we have calculated the gaps below the largest eigenvalue of $\log (V)$ for "excitations" with wavenumbers $\omega=0$ and $\omega=\pi$, at multiple temperatures. This gap is equal to $L / \xi$, where $\xi$ is the "ferromagnetic" (for $\omega=0$ ) or "antiferromagnetic" (for $\omega=\pi$ ) correlation length. Each sample shows one or more phase transitions where one of these correlation lengths diverges, so the corresponding gap vanishes. Because the model is a planar Ising model that is in the free-fermion class, we expect that all the phase transitions are of the usual two-dimensional Ising model universality class. Our results do appear to be consistent with this expectation, although we have not made thorough 
checks. The model is periodic and in its ordered phases has correlations that are either ferromagnetic or antiferromagnetic between equivalent spins in adjacent unit cells along the rows. Within a unit cell the ordering is into a spin-glass magnetization pattern determined by the random couplings and the temperature. When a ferromagnetic phase transition occurs, the gap at $\omega=0$ vanishes, while that at $\omega=\pi$ vanishes at an antiferromagnetic transition. Note, we define the wavenumber $\omega$ in terms of the unit cell size, so $\omega=\pi$ corresponds to a period of two unit cells along the rows. For a number of samples we have also examined the gaps at other wavenumbers; these gaps are never found to vanish. Because we have chosen the unit cells to be vertically reflection-invariant, the correlations between equivalent spins in adjacent unit cells in different (horizontal) rows are always ferromagnetic (the eigenvalues of the transfer matrix $V$ are all real and positive).

An example of the most common type of sample, with just one phase transition, is shown in Fig. 1. The phase transition is clearly seen as the point where the gap at $\omega=\pi$ vanishes. For this sample the ground state is antiferromagnetic between unit cells (although samples with ferromagnetic ground states are equally common), and the interaction between the unit cells remains antiferromagnetic for temperatures below the phase transition. However, the net interaction between unit cells may change sign with changing temperature as the balance between energetic and entropic interactions is changed in the full free energy. For the sample of Fig. 1 this happens in the disordered high temperature phase, near $1 / T=1.2$.

In some samples (between 10 and $20 \%$ of the samples for $20 \leq L \leq 40$ ) the sign of the intercell interaction changes at low enough temperature to produce multiple phase transitions. An example is shown in Fig. 2. Here the ground state is again antiferromagnetic, but the intercell interaction changes sign at low temperature (near $1 / T=3$ ). This low temperature transition region is shown with expanded scales in Fig. 3. There is a narrow interval of disordered phase where the interaction between horizontally-adjacent unit cells in a row is very small or zero. Note that the minimum gap remains less than about 0.1 in this low-temperature disordered phase. This means the vertical correlation length always exceeds about 10 unit cells, which is 300 nearest-neighbor spacings in this case. Thus this is a very well correlated disordered phase, as is to be expected at such a low temperature. This disordered phase is bounded below by the low-temperature antiferromagnetic phase and above by an intermediate-temperature ferromagnetic phase. The ferromagnetic phase disorders in the third phase transition at higher temperature (Fig. 2). We have seen samples that show as many as 5 transitions in the temperature range we studied, but these are rather rare (roughly 40 in a total population of about 14,000 samples examined).

Our system should behave like a spin glass as long as the correlations between unit cells are weak. However, once the correlation length reaches the unit cell size $L$ the system "realizes" that it is periodic and can order as a regular two-dimensional Ising model. The temperature, $T_{c}$, of the first phase transition met on lowering the temperature thus indicates where the correlation length is of order $L$. The correlation length in the two-dimensional spin glass diverges as $T^{-\nu}$ at low temperature. [1.2] Thus we expect the mean or median transition temperature for our periodic approximations to the spin glass to decrease with increasing $L$ as $L^{-1 / \nu}$. For a small minority of samples the transition is at a lower temperature than we studied, so we have only an upper bound on it. Because of this we do not have a proper measurement of the mean $T_{c}$, but we do have the medians for each size, $T_{\text {med }}(L)$. The results are $T_{\text {med }}(20)=0.757 \pm 0.003$ from 5000 samples, $T_{\text {med }}(30)=0.654 \pm 0.003$ from 5000 samples, and $T_{\text {med }}(40)=0.588 \pm 0.003$ from 4000 samples. These give an estimate of $\nu \cong 2.7$ from fitting to $T_{\text {med }}(L) \sim L^{-1 / \nu}$. The distribution of $T_{c} / T_{\text {med }}(L)$ should be independent of $L$, by scaling. This is demonstrated in Fig. 4 , where the histograms of the scaled $T_{c}$ are shown.

Previous estimates of $\nu$ for the two-dimensional Ising spin glass with a continuous distribution of bond strengths have ranged from near 2.0, 迥 8 ] to close to 4 , [7] with the estimates depending on the scaling assumptions used to obtain them. Our estimate of 2.7 is comfortably within this range. Note that we have used data for only a factor of two change in $L$ for this estimate, so it could be substantially affected by corrections to scaling that we would not detect. For smaller $L$ (e.g., $L=10$ ) we saw clear deviations from scaling, for example in the shape of the mean gap vs. $T$ (Fig. 5), so we have not used the smaller $L$ data. The unusual distribution of interactions we use is a sort of hybrid of the $\pm \mathrm{J}$ and continuous distributions, so could show some sort of crossover at short length scales.

Different researchers using the same assumptions have generally good agreement on the value of $\nu$ for Gaussiandistributed interactions, so the differences do not appear to arise from variable quality in the underlying numerical data. The wide variation of estimates of $\nu$ indicates that there is something about the low-temperature scaling in the two-dimensional Ising spin glass that is not yet understood.

We have also averaged the minimum of the gaps (at $\omega=0$ or $\pi$ ) over all samples for each $L$ and various temperatures. The gap is $L / \xi$, so is dimensionless, and thus its average should be independent of $L$ at a fixed $T / T_{m e d}(L)$. The resulting scaling plot of the average gap is shown in Fig. 5. The scaling collapse is quite good near and below $T_{m e d}$, but clear deviations from scaling can be seen at higher temperatures. 


\section{IV. "CHAOS"}

The term "chaos" is used to refer to the extreme sensitivity of the sign of the long-distance spin-spin correlations to changes of temperature in a given spin glass sample. [10] One manifestation of these sign changes that we have already discussed is the multiple phase transitions that occur in some samples. To make a more quantitative study of this we define the coupling between adjacent unit cells to be ferro- or antiferromagnetic based on whether the gap is smaller at $\omega=0$ or $\omega=\pi$, respectively. Then we record each time this coupling changes sign as the temperature is changed for each sample. Let $n_{L}(T)$ be the number of sign changes per sample between zero temperature and temperature $T$ for unit cell size $L$. We do not directly measure $n_{L}(T)$, since we do not follow the samples all the way to $T=0$, but we do measure the change in $n_{L}(T)$ over the temperature range we study. In Fig. 6 we show $n_{L}(T)-n_{L}\left(T_{\min }\right)$ vs. $T$, where $T_{\min }$ is the lowest temperature we examined for each size $L$. It is clear from this figure that the number of coupling changes increases with increasing $L$.

Why does the coupling change sign with changing $T$ ? This occurs because for nonzero $T$ and large enough $L$ the free energy difference between ferro- and antiferromagnetic spin patterns is a near-cancellation of larger entropy and energy differences. The free energy difference varies with $L$ as $\Delta F \sim L^{-1 / \nu}$, so actually decreases with increasing $L$ in this two-dimensional system. At low temperatures, $T<<T_{c}$, the difference between the two patterns is typically a relative domain wall running across the full unit cell. The two different spin configurations along that domain wall will have randomly different local entropies due to fluctuations at small length scales. Thus it has been argued [9, 10] that the entropy difference between the two patterns grows with $L$ as $\Delta S \sim L^{d_{s} / 2}$, where $d_{s}$ is the fractal dimension of the relative domain wall. For this case $1 \leq d_{s} \leq 2$, the lower limit being that of a straight wall, and the upper limit being a space-filling rough wall. Since the free energy difference is $\Delta F=\Delta E-T \Delta S$, where $\Delta E$ is the energy difference, the typical temperature change needed to change the sign of $\Delta F$ varies with $\mathrm{L}$ as $\delta T \sim \Delta F / \Delta S \sim L^{-d_{s} / 2-1 / \nu}$. Thus the rate of sign changes with changing $T$ is expected to increase strongly with increasing $L$, as we show in Fig. 6 . We believe this is the first direct measurement of these sign changes of the correlations in a realistic spin glass model at low temperatures.

The above argument applies at low temperatures where the system is well-correlated within a unit cell, so it is reasonable to assume the difference between the two spin patterns is simply a relative domain wall. This picture will break down at higher temperatures as $T$ approaches and exceeds $T_{c}$. To take account of this, a naive finite-size scaling hypothesis is

$$
n_{L}(T) \sim L^{\zeta} T_{\text {med }}(L) N\left(\frac{T}{T_{\text {med }}(L)}\right),
$$

where $N$ is a scaling function, and the chaos exponent $\zeta$ determines how the scaled chaos increases with increasing $L$. In Fig. 7, we show the data scaled in this way, with $\zeta=0.85$. As with our estimate of $\nu$, this estimate of $\zeta$ is from scaling over a factor of only two in length. There are presumably errors in these exponent estimates due to corrections to scaling, which are likely to be much larger than those due to the statistical uncertainties in our data. Thus we do not know how large an error estimate to quote for our exponent estimates. Our estimate of $\zeta \cong 0.85$ is consistent with the $\zeta=1.0 \pm 0.2$ found by Ney-Nifle and Young [8] from overlaps between different temperatures fit to a similar scaling form. In the latter Monte-Carlo study the overlap was only suppressed by a few per cent by the temperature change, indicating that only a very small fraction of the spins were flipped by the temperature changes in the rather small $(L \leq 10)$ samples they could study using that approach. In contrast, we are able to access substantially larger length scales and a wider temperature range, and find at least one sign change in the correlations for a large fraction of the samples.

[1] A. J. Bray and M. A. Moore, J. Phys. C 17, L463 (1984).

[2] W. L. McMillan, Phys. Rev. B 30, 476 (1984).

[3] R. N. Bhatt and A. P. Young, Phys. Rev. B 37, 5606 (1988).

[4] N. Kawashima and M. Suzuki, J. Phys. A 25, 1055 (1992).

[5] N. Kawashima, N. Hatano and M. Suzuki, J. Phys A 25, 4985 (1992).

[6] S. Liang, Phys. Rev. Lett. 69, 2145 (1992).

[7] H. Rieger, et al., J. Phys. A 29, 3939 (1996).

[8] M. Ney-Nifle and A. P. Young, J. Phys. A [in press].

[9] D. S. Fisher and D. A. Huse, Phys. Rev. Lett. 56, 1601 (1986); Phys. Rev. B 37, 373 and 386 (1988). 
[10] A. J. Bray and M. A. Moore, Phys. Rev. Lett. 58, 57 (1987).

[11] L. Onsager, Phys. Rev. 65, 117 (1944).

[12] B. Kaufman, Phys. Rev. 76, 1232 (1949).

[13] L.-F. Ko and M. E. Fisher, J. Stat. Phys. 58, 249 (1990).

[14] D. B. Abraham, L.-F. Ko and N. M. Svrakić, J. Stat. Phys. 56, 563 (1989).

[15] B. Haible, "CLN, a class library for numbers", available from ftp://ma2s2.mathematik.uni-karlsruhe.de/pub/gnu/cln/tar.z (1996).

[16] L.-F. Ko and M. E. Fisher, (to be published).

\section{FIGURE CAPTIONS}

FIG. 1. The gaps below the highest state in the spectrum of the logarithm of the transfer matrix for various wavenumbers vs. the inverse temperature $(1 / T)$ for a specific sample with unit cell size $L=30$. At low temperature, this sample orders antiferromagnetically, as indicated by the gap at $\omega=\pi$ vanishing at the phase transition. However, the minimum gap moves to $\omega=0$ at the highest temperature point shown.

FIG. 2. As in Fig. 1, the gap vs. $1 / T$, but here for a different specific sample with $L=30$ that instead shows three phase transitions. Moving from left to right (high to low temperature) the phases are: disordered, ferromagnetic, disordered again, and antiferromagnetic. The intercell interaction changes sign near $1 / T=3$ from ferromagnetic at higher temperature to antiferromagnetic at lower temperature.

FIG. 3. The lower-temperature transition region in Fig. 2, shown with expanded scales.

FIG. 4. The histograms of the scaled transition temperatures, $T_{c} / T_{\text {med }}(L)$ for each size $L$, where $T_{\text {med }}(L)$ is the median transition temperature for unit cell size $L$. The bins in these histograms are all of width 0.1 along the horizontal axis, and the fraction of the samples lying within each bin is indicated.

FIG. 5. The mean of the gap below the highest state in the spectrum of the logarithm of the transfer matrix vs. the temperature scaled by the median transition temperature, $T_{m e d}(L)$, for each size $L$.

FIG. 6. The number of sign changes in the correlations between adjacent unit cells per sample between the lowest temperature studied, $T_{\text {min }}$, and temperature $T$. For sizes $L=20,30,40$ we used $1 / T_{\min }=3.4,3.8,4.2$, respectively.

FIG. 7. The number of sign changes per sample, scaled according to Eq. (4.1), with $\zeta=0.85$. 